\title{
Identification and rejection of fake reconstructed jets from a fluctuating heavy ion background in ATLAS
}

\author{
N. Grau $^{\mathrm{a}}$ \\ on behalf of the ATLAS Collaboration \\ Columbia University, New York, NY, USA
}

Received: 7 October 2008 / Published online: 10 March 2009

(c) Springer-Verlag / Società Italiana di Fisica 2009

\begin{abstract}
Full jet reconstruction in relativistic heavy ion collisions provides new and unique insights to the physics of parton energy loss. Because of the large underlying event multiplicity in $A+A$ collisions, random and correlated fluctuations in the background can result in the reconstruction of fake jets. These fake jets must be identified and rejected to obtain the purest jet sample possible. A large but reducible fake rate of jets reconstructed using an iterative cone algorithm on HIJING events is observed. The absolute rate of fake jets exceeds the binary-scaled $p+p$ jet rate below $50 \mathrm{GeV}$ and is not negligible until $100 \mathrm{GeV}$. The variable $\Sigma j_{T}$, the sum of the jet constituent's $E_{T}$ perpendicular to the jet axis, is introduced to identify and reject fake jets at by a factor of 100 making it negligible. This variable is shown to not strongly depend on jet energy profiles modified by energy loss. By studying azimuthal correlations of reconstructed di-jets, the fake jet rate can be evaluated in data.
\end{abstract}

\section{Introduction}

Complete jet reconstruction in relativistic heavy ion collisions is essential for a direct measurement of the energy lost by a high- $p_{T}$ colored parton traversing the colored medium produced in these collisions. Measurements of such energy loss have been made from experiments at Brookhaven National Laboratory's (BNL) Relativistic Heavy Ion Collider (RHIC) where single particle production rates in $\sqrt{s_{N N}}=$ $200 \mathrm{GeV} \mathrm{Au}+\mathrm{Au}$ collisions are suppressed relative to binary-collision scaled $p+p$ rates at the same $\sqrt{s}$ [1]. These measurements alone, however, are insufficient to constrain the details of the energy loss mechanism. For example, the

\footnotetext{
a e-mail: ncgrau@nevis.columbia.edu

All results presented use modified versions of ATLAS software and should be considered "ATLAS preliminary".
}

current data cannot yet be used to determine the relative contributions of radiative and collisional enery loss, or even determine whether jet quenching is a perturbative or nonperturbative process. Because of the large underlying event multiplicity, $\mathrm{d} N / \mathrm{d} \eta \sim 600$ for the top $10 \% \mathrm{Au}+\mathrm{Au}$ collision cross section [2], direct jet reconstruction at RHIC is difficult, though attempts are underway [3,4]. As a proxy for (di-)jet reconstruction, two, high- $p_{T}$ particle azimuthal correlations have been measured. Results from two-particle correlations reveal that the trigger jet is essentially unmodified while the recoil jet is strongly suppressed [5], further lending support to the idea that parton energy loss is the mechanism of the suppression. However, two-particle correlations have not revealed expected modifications to jet properties [6] such as softening of the fragmentation function [7], broadening of the di-jet acoplanarity [8], etc. expected from energy loss models that reproduce the single particle suppression. One possible reconciliation between the single particle and two-particle results is that the two-particle results are "energy loss biased". That is, by requiring that two high- $p_{T}$ particles be present, events are chosen where the jet loses little, if any, energy. This can occur when the jet traverses a short path length [9] or emits no gluons due to fluctuations in radiation (punchthrough).

Direct measurements of jets reconstructed via standard algorithms employed in $p+\bar{p}(p)$ or $e^{+} e^{-}$collisions modified to handle the underlying heavy ion event, should reduce such energy loss biases. If the energy loss is dominated by gluon bremsstrahlung radiated predominantly inside the jet cone [10], the lost energy will be recovered by reconstructing the full jet profile. If, however, energy loss is nonperturbative [11] or radiates energy outside of the cone [12], a suppressed jet rate compared to binary-scaled $p+p$ will be observed. Whatever the scenario, direct measurements of jets will make a significant impact in understanding parton energy loss. 
In the near future the Large Hadron Collider (LHC) at CERN will deliver collisions of $\mathrm{Pb}+\mathrm{Pb}$ at $\sqrt{s_{N N}}=5.5 \mathrm{TeV}$. At this energy and nominal delivered luminosity, 20 million jets with $E_{T}>50 \mathrm{GeV}$ will be produced per month of $\mathrm{Pb}+$ $\mathrm{Pb}$ running [13]. These jets should be visible above the large underlying event multiplicity and can be reconstructed by jet reconstruction algorithms appropriately modified to handle the underlying event.

The ATLAS experiment will carry out extensive measurments of jet production in heavy ion collisions using its nearly hermetic electromagnetic and hadronic calorimeters and its large acceptance silicon tracking system. Techniques have been developed for iterative cone jet reconstruction in heavy ion collisions in ATLAS [14]. These techniques require subtraction of the underlying event energy within the calorimeter prior to running the iterative cone jet algorithm (see Sect. 3). Fluctuations in the background remain after subtraction and a stable cone, defined by the algorithm, can be obtained from random or correlated background. In studying any aspect of jets and their modification, such fake jets must be tagged and removed from the data sample. It is, therefore, necessary to study the characteristics of fake jets so as to extract a sample of jets with high purity.

This article describes the techniques that have been developed to identify and reject fake jets with the ATLAS detector arising from fluctuations in the underlying heavy ion event. The article is outlined as follows. In Sect. 2 the relevant aspects of the ATLAS detector are defined. Section 3 presents the necessary details of jet reconstruction in heavy ion collisions in ATLAS and the identification of fake jets. This is followed by Sect. 4 where the results of fake jet detection are presented. A discussion of potential biases associated with the method of rejection and evaluation of rejection with data is given in Sect. 5 .

\section{The ATLAS detector}

The ATLAS detector $[15,16]$ is a large, multi-purpose, high energy physics experiment designed to detect rare processes in $p+p$ collisions at $\sqrt{s}=14 \mathrm{TeV}$ at the LHC. ATLAS has also been shown to be an excellent detector for measurements of both high $p_{T}$ and soft observables in heavy ion collisions [17]. The jet reconstruction described here is purely calorimetric. The ATLAS calorimeter is largely divided into a liquid argon (LAr) barrel electromagnetic calorimeter covering $|\eta|<3.2$, a LAr forward hadronic calorimeter covering $3.2<|\eta|<5$ and a barrel tile hadronic calorimeter covering $|\eta|<1.5$. Each calorimeter compartment is divided into longitudinal sections providing energy readout at different depths in the calorimeter. The barrel electromagnetic calorimeter is divided into 3 layers. The front "strip" layer, with approximately $6 X_{0}$, has a typical cell (single readout) segmentation of $\Delta \eta \times \Delta \phi$ of $0.003 \times 0.1$. The middle layer, with $24 X_{0}$, has a cell size of $0.025 \times 0.025$ in $\Delta \eta \times \Delta \phi$. The barrel tile calorimeter has three layers with depth-dependent cell size; the first layer has $0.1 \times 0.1 \mathrm{seg}$ mentation. For reconstructing jets, towers in a fixed grid of $0.1 \times 0.1$ in $\Delta \eta \times \Delta \phi$ are built from sums of cell energies in all layers.

\section{Jet reconstruction and fake jet identification}

To evaluate the expected jet reconstruction performance in heavy ion collision with the ATLAS detector, di-jet PYTHIA [18] events were embedded in HIJING [19] heavy ion events. The HIJING events (referred to as "unmodified") were generated without jet quenching and without a maximum $p_{T}$ cut on the hard scattering processes. The unmodified events result in correlated and fluctuating backgrounds from (mini-)jets, $c-\bar{c}$ and $b-\bar{b}$ production, and longitudinal string radiation all unsuppressed from the lack of quenching. The HIJING events containing embedded jets were propagated through the ATLAS detector using the GEANT4 software package, digitized to resemble raw data, and then processed by the standard ATLAS offline software chain.

Jet reconstruction was performed using a seeded iterative cone jet algorithm [20,21] with a seed tower energy of $5 \mathrm{GeV}$ and a cone radius $R=\sqrt{\Delta \phi^{2}+\Delta \eta^{2}}=0.4$. On average, in unmodified HIJING events with $\mathrm{d} N / \mathrm{d} \eta=2700$, $150 \mathrm{GeV}$ of background event $E_{T}$ is contained within the cone. The method used to subtract the background is to

1. find regions of high energy in the calorimeter (potential jet regions)

2. calculate the average background $E_{T}$ in the calorimeter for each longitudinal layer $i$ and as a function of $\eta$, $E_{T}^{\mathrm{bkgr}}(i, \eta)$, excluding the regions found in step 1

3. subtract $E_{T}^{\mathrm{bkgr}}(i, \eta)$ from all cells

4. build the $0.1 \times 0.1$ grid of calorimeter towers from these modified cells.

The subtracted towers from this procedure are provided as input to the seeded cone jet algorithm.

Figure 3.1 shows the invariant yields of jets in $b=2$ fm, $\mathrm{d} N / \mathrm{d} \eta=2700$, HIJING events. The solid (blue) circles indicates the binary-scaled $p+p$ jet rate obtained from PYTHIA. The diamonds show the reconstructed spectrum, including embedded PYTHIA jets and fake jets which survive fake jet rejection (see Sect. 4). The reconstructed spectrum is uncorrected for efficiency, energy scale, and energy resolution. The need for these corrections is clear below $70 \mathrm{GeV}$ while the reconstructed and expected spectra agree well above $70 \mathrm{GeV}$. For these high-multiplicity HIJING events, $70 \mathrm{GeV}$ jets have a reconstruction efficiency 
of $70 \%$, an energy scale offset of $-2.5 \%$, and an energy resolution of $25 \%$. A complete evaluation of the centrality and $E_{T}$-dependence of the reconstruction is given in Ref. [14].

Fake jets were produced by generating, simulating in ATLAS, and performing jet reconstruction on a second set of HIJING events, labeled "hard-cut". These events differ from the unmodified HIJING events in that the parton spectrum at $Q^{2}>100 \mathrm{GeV}^{2}$ is not sampled. Therefore, jets with $E_{T}>$ $10 \mathrm{GeV}$ are purposefully suppressed with the intent being that any jet with $E_{T} \gg 10 \mathrm{GeV}$ is a fake jet. Even with a hard cut, jets above $10 \mathrm{GeV}$ can occur from initial and/or final state radiation. What was also found was that longitudinal strings can radiate into midrapidity resulting in $10^{-2}$ jets with $E_{T}>50 \mathrm{GeV}$ per event. These were removed from

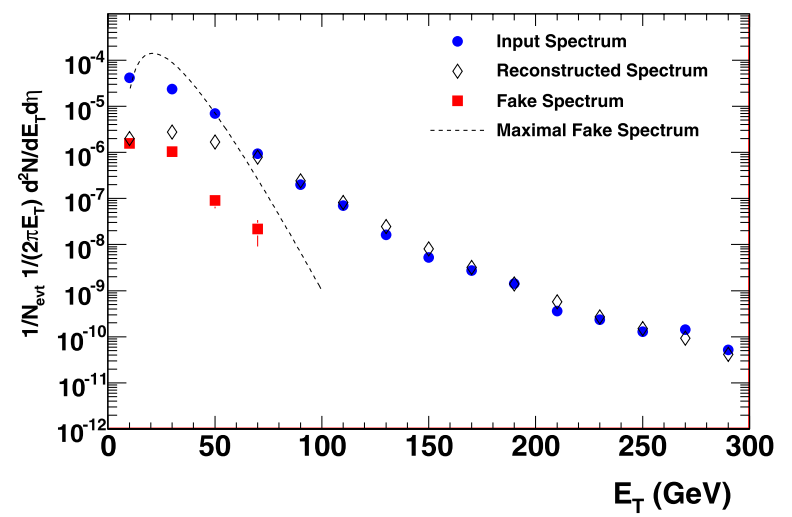

Fig. 3.1 Comparison of the spectrum of jets in $\mathrm{d} N / \mathrm{d} \eta=2700$, $b=2 \mathrm{fm}$, HIJING events. The (blue) filled circles are the expected spectrum from binary-scaled $p+p$, the diamonds are the raw reconstructed spectrum uncorrected for jet reconstruction efficiency and energy resolution. The dashed line indicates the raw fake rate evaluated from hard-cut HIJING events. The (red) squares indicate the irreducible fake spectrum after rejection of the fake jets

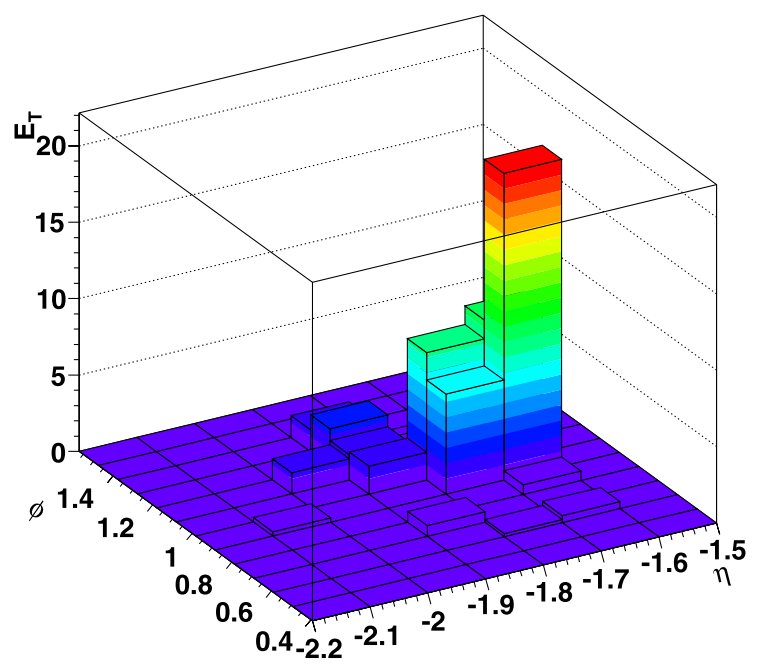

the fake jet sample by excluding jets which match a HIJING parton above an appropriate threshold.

The resulting reconstructed jets from the hard-cut HIJING events then define the "maximal" fake rate plotted in Fig. 3.1. The rate of fake jets exceeds that of the expected jet yield at low- $E_{T}$. The ratio of fake to reconstructed yields is 4.2 at $50 \mathrm{GeV}, 0.33$ at $70 \mathrm{GeV}$, and 0.03 at $90 \mathrm{GeV}$. This shows that the contribution of fake jets decreases dramatically with reconstructed jet $E_{T}$. However, if it is desired to measure jets at moderate $E_{T}$, a rejection procedure is needed.

It is possible that the fake rate is overestimated. Part of the hard particle production in HIJING results from longitudinal strings, color fields between the nuclei, which, because of their large energy at the LHC, can fragment into a jet at midrapidity. Although an attempt was made to remove such jets from the hard-cut events, a sample of hard-cut HIJING events with the longitudinal string radiation suppressed was also studied. The maximal rate was reduced such that the ratio of the fake to reconstructed yield is 1.5 at $50 \mathrm{GeV}, 0.11$ at $70 \mathrm{GeV}$, and 0.01 at $90 \mathrm{GeV}$. The longitudinal radiation, however, did not change the characteristics of the fake jets that were produced, nor their subsequent identification and rejection.

\section{Fake jet rejection}

Rejection of fake jets is maximized when exploiting the differences between the embedded and fake jets. Figure 4.1 shows a general feature of fake jets by comparing two jets with the same reconstructed jet $E_{T}(=57 \mathrm{GeV})$. The jet in the left panel is an embedded PYTHIA jet and the jet in the right panel is reconstructed from the hard-cut HIJING

Fig. 4.1 Reconstructed jets with $E_{T}=57 \mathrm{GeV}$ from PYTHIA embedded into unmodified HIJING (left) and from hard-cut HIJING (right). The jet from the hard-cut HIJING is fake since no jets of this energy should exist in this sample (see text) 
events. Even though the fragmentation of the PYTHIA jet is not uniform in $\eta$ and $\phi$, a clear core is seen. The fake jet from the hard-cut HIJING event is a cone which stabilized around three towers at large angle to the reconstructed jet axis and no core exists. Such a structure is generally indicative of the jets from the hard-cut HIJING events: little to no core and energy at large angles from the jet axis.

The variable used to discriminate against fake jets is $\Sigma j_{T}$, which is constructed to give larger weight to energies at large angle with respect to the jet direction. It is defined as

$$
\Sigma j_{T}=\sum_{\text {cell } \in \text { jet }} E_{T, \text { cell }} \sin R_{\text {cell }}
$$

where $E_{T \text {,cell }}$ is the $E_{T}$ with respect to the beam and $R_{\text {cell }}$ is the angle (in $\phi$ and $\eta$ ) of the cell with respect to the jet. It has a trivial jet $E_{T}$ dependence. The definition can be rearranged to yield

$$
\Sigma j_{T}=\langle\sin R\rangle E_{T, \mathrm{jet}}
$$

where $\langle\sin R\rangle=\Sigma_{\text {cell }} E_{T, \text { cell }} \sin R_{\text {cell }} / E_{T, \text { jet }}$ is the energy weighted average of $\sin R$. Therefore, broad distributions would have a larger $\Sigma j_{T}$ than narrow distributions at the same $E_{T}$. Figure 4.2 shows the jet $E_{T}$ dependence of $\Sigma j_{T}$. It is linear for the fake jets measured from hard-cut HIJING events. A rising trend is also observed in the embedded jets, but, because jets become more collimated with increasing $E_{T}$, $\langle\sin R\rangle$ decreases with increasing $E_{T}$, and, therefore, the rising trend of $\Sigma j_{T}$ increases more slowly at high $E_{T}$. This has the effect that the discrimination between real and fake jets becomes more effective with increasing jet $E_{T}$.

Because of the $E_{T}$ dependence of $\Sigma j_{T}$, the applied cut is an $E_{T}$-independent quantity: the number of RMS from the fake-jet mean, defined as

$\sigma_{\Sigma j_{T}}=\frac{\Sigma j_{T}-\left\langle\Sigma j_{T}\right\rangle\left(E_{T}\right)}{\sigma\left(E_{T}\right)}$

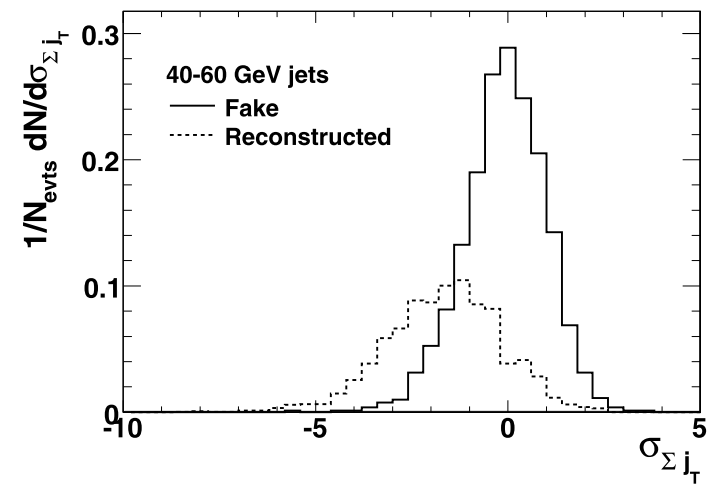

where $\left\langle\Sigma j_{T}\right\rangle\left(E_{T}\right)$ and $\sigma\left(E_{T}\right)$ are the $E_{T}$-dependent mean and RMS, respectively, fitted to the hard-cut HIJING jets from Fig. 4.2. Figure 4.3 shows the distribution of $\sigma_{\Sigma j_{T}}$ for two different jet $E_{T}$ bins for embedded and fake jets. By definition the fake jets have a mean of zero and an RMS of 1 while the embedded jets have an obviously smaller mean in this variable. The rate of fakes compared to reconstructed embedded jets is also seen in these plots as well as its strong $E_{T}$ dependence.

Because of the clear separation between the embedded and fake jets, a cut can be applied to reject fake jets at a desired level. However, since the distributions overlap, there is a trade-off between fake jet rejection and efficiency loss. For example, the cut applied in Fig. 3.1 is to reject jets with $\sigma_{\Sigma j_{T}}>-2.5$. For both $E_{T}$ bins shown in Fig. 4.3 this cut is not only effective in rejecting nearly all fake jets but also results in a sizeable loss of real jets. Figure 4.4 shows the anti-correlation between the rejection of fake jets and the re-

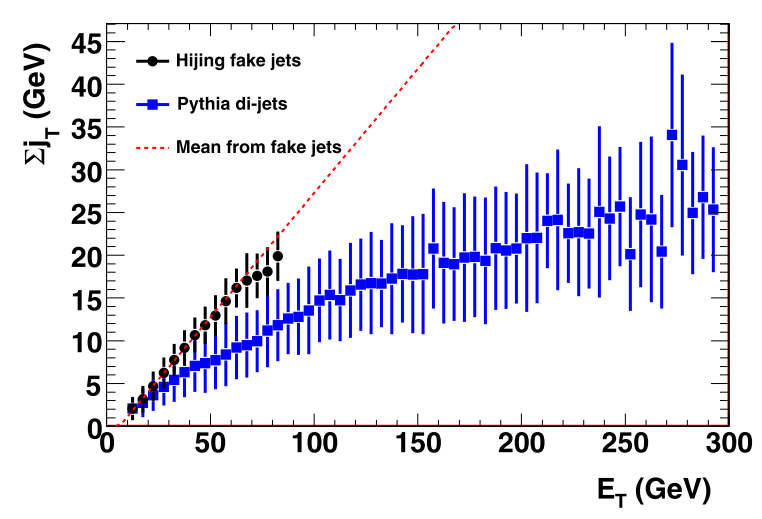

Fig. 4.2 The reconstructed jet $E_{T}$ dependence of $\Sigma j_{T}$ for fake jets from the unmodified HIJING sample (circles) and for reconstructed embedded jets in $\mathrm{d} N / \mathrm{d} \eta=2700$ (squares). The bars indicate the root-mean-square of the distribution in each $E_{T}$ bin. The dashed line is a fit to the HIJING fake jets. A clear separation between the fake jets and embedded jets is seen over all $E_{T}$. The larger $\Sigma j_{T}$ for the fake jets indicates that they have a broader energy distribution than the embedded jets

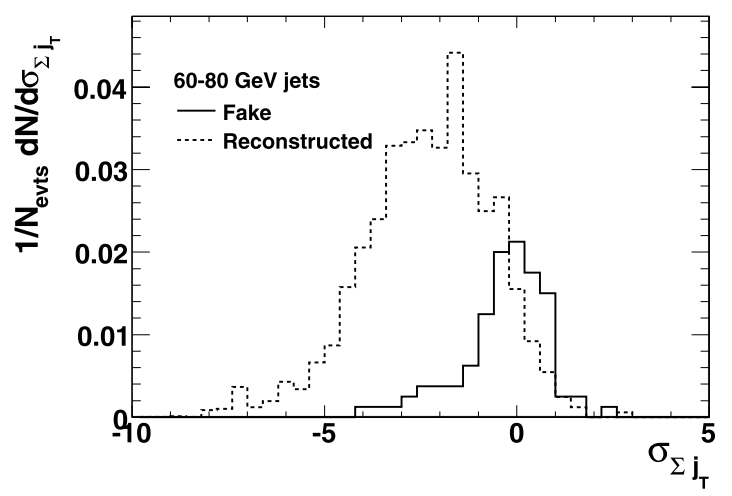

Fig. 4.3 The jet $E_{T}$-independent cut variable $\sigma_{\Sigma j_{T}}$, the number of $\sigma$ from the fake jet mean (see (4.3)) for two different jet $E_{T}$ bins $40-60 \mathrm{GeV}$ (left) and 60-80 GeV (right). There is clear separation between the embedded jets (dashed line) compared to the fake jets (solid line) 


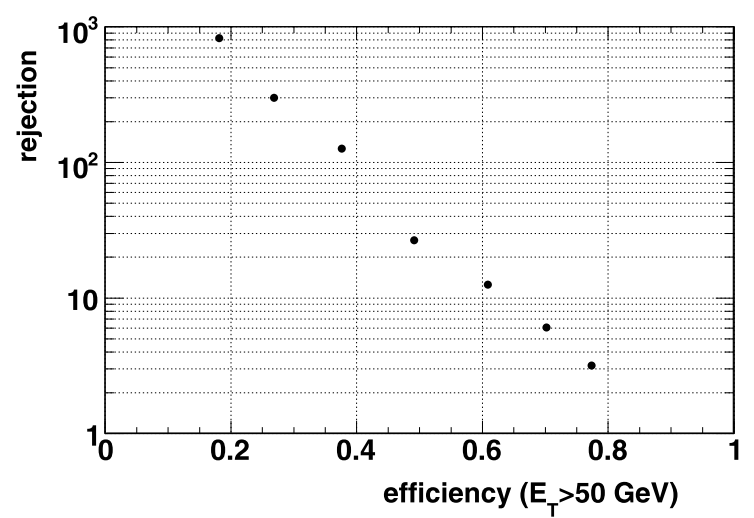

Fig. 4.4 Fake jet rejection vs jet reconstruction efficiency. Each point represents a different cut in $\sigma_{\Sigma j_{T}}$ from rejecting $\sigma_{\Sigma j_{T}}>-1$ (lower right) to $\sigma_{\Sigma j_{T}}>-4$ (upper left)

construction efficiency of embedded jets. In the end the statistics and the desired purity of the data sample will dictate what cut will be applied in real data (see Sect. 5.2).

\section{Results and discussion}

The variable $\Sigma j_{T}$ has been shown to be effective at rejecting fake, reconstructed cone jets from HIJING events. In this section the effect of energy loss on the rejection and evaluation of the fake jet rate with real data are discussed.

\subsection{Effect of energy loss}

In radiative energy loss, the energy profile of a given jet is modified jet-by-jet, and on average the energy profile is expected to show large modification [22]. Because the $\Sigma j_{T}$ is a shape cut, it is possible that such a cut could bias results from measuring the modification of the energy profile of a jet.

An estimate of the effect can be obtained using (4.2) and assuming a Gaussian distribution of energy within a jet. For a Gaussian with an RMS of $\sim 0.2$, then $\langle\sin R\rangle \sim\langle R\rangle$. At a fixed jet $E_{T}$ the difference between embedded and fake $\left\langle\Sigma j_{T}\right\rangle$ then is directly related to the difference between $\langle R\rangle$. From Fig. 4.2, at $50 \mathrm{GeV}$ the fake jet $\left\langle\Sigma j_{T}\right\rangle$ is twice that of the embedded jet. Therefore, $\langle R\rangle$ of the fake jet is twice that of the embedded jet. This is quite a large change for a jet with an RMS of 0.2 to one with 0.4. Such a difference between vacuum and medium modified shape may be expected for $50 \mathrm{GeV}$ gluon jets [22].

An attempt at studying the energy loss effect on the rejection of $\Sigma j_{T}$ has been done by simulating and reconstructing a set of PYQUEN [23] jets in the ATLAS detector. PYQUEN is a set of routines which modifies PYTHIA partons by energy loss and including the radiated gluons prior to fragmentation. The events were generated assuming $b=0$ fm and they were not embedded into HIJING. Figure 5.1 shows the ratio of $\left\langle\Sigma j_{T}\right\rangle$ as a function of $E_{T}$ (i.e. Fig. 4.2) for quenched to unquenched jets (circles) and for hard-cut HIJING jets to embedded jets (squares). Little change between the quenched and unquenched jets is observed in contrast to the large difference between the fake and embedded $\left\langle\Sigma j_{T}\right\rangle$.

The jet energy profile in PYQUEN jets are modified, but not to the same extent as those predicted in Ref. [22], leading to much less change in $\langle R\rangle$. These results were produced after a simulation through the ATLAS calorimeter. It is possible that the segmentation is also coarse enough to smear any effect generated by PYQUEN. It should be clear, however, that because $\Sigma j_{T}$ is a shape variable, it may show different sensitivities to different jet profiles. The final resolution of how sensitive must wait for analysis of real data.

\subsection{Rejection in data}

Determining the absolute rate of fake jets in real data, regardless of the rejection variable, can be done by studying the $\Delta \phi$ distribution of the pairs of reconstructed jets. This is a useful variable because 1) the position resolution of jets is good, 2) it has a clear signal peak at $\Delta \phi=\pi$, and 3) fake jets will be randomly associated with other real or fake jets in the events and result in a pedestal. The rejection variable distribution should be different for different regions of $\Delta \phi$.

This is the case for $\sigma_{\Sigma j_{T}}$. Figure 5.2 shows the distribution of all jets pairs with $E_{T}^{A}>60 \mathrm{GeV}$ correlated with with $E_{T}^{B}>40 \mathrm{GeV}$ with different $\sigma_{\Sigma j_{T}}$ cuts applied. For the successively hard $\sigma_{\Sigma j_{T}}$ cuts the pedestal is suppressed. The stars indicate the cut used in Fig. 3.1 which removes a significant fraction of fake jets. With the tightest cut, the signal peak at $\Delta \phi=\pi$ becomes slightly narrower. This may indicate that the hardest cut removes the radiated jets in a 3 -jet event.

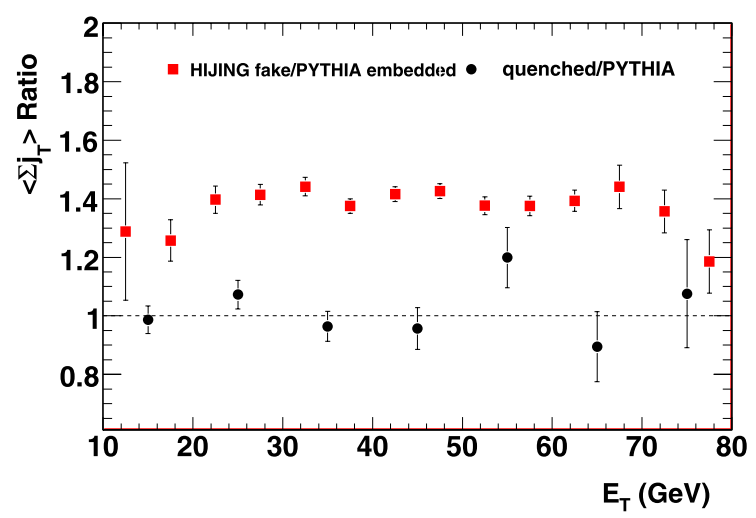

Fig. 5.1 The $E_{T}$-dependent ratio of $\left\langle\Sigma j_{T}\right\rangle$ in PYQUEN jets to embedded jets (black circles) compared to fake HIJING jets to embedded jets (red squares) (from Fig. 4.2). No difference is observed between the quenched and unquenched jets and it is much less than the difference between the fake and embedded jets 


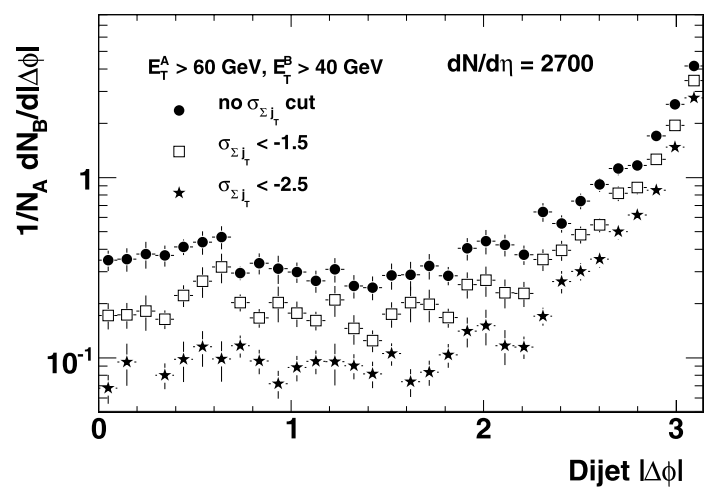

Fig. 5.2 $\Delta \phi$ distribution between reconstructed jets with $E_{T}^{A}>$ $60 \mathrm{GeV}$ and $E_{T}^{b}>40 \mathrm{GeV}$ for different $\sigma_{\Sigma j_{T}}$ cuts

\section{Summary}

A large fake rate of cone jets from HIJING events is observed, exceeding the expected $p+p$ jet rate at low $E_{T}$. They result from stable cones being fitted to random and correlated background fluctuations. The variable $\Sigma j_{T}$ has been used to identify and reject these fake jets. It is shown that this variable is insensitive to the effects of energy loss on jets. It is conceivable that neither the extreme fluctuations nor the characteristics of fake jets (broader, large angle energy distribution) generated in HIJING are observed in the data. In fact, the fake rate will strongly depend on the model of the background and, indeed, will require measurement in the data itself. Even so, because correlated and random fluctuations can produce stable cone jets, it is necessary for any jet analysis in heavy ion collisions at RHIC or the LHC to demonstrate a lack of contamination of such jets.

\section{References}

1. K. Adcox et al. (PHENIX Collaboration), Phys. Rev. Lett. 88, 022301 (2002)

2. B.B. Back et al. (PHOBOS Collaboration), Phys. Rev. C 65, 061901 (2002)

3. J. Putschke (STAR Collaboration), arXiv:0809.1419 [nucl-ex]

4. Y.S. Lai, B.A. Cole, arXiv:0806.1499 [nucl-ex]

5. C. Adler et al. (STAR Collaboration), Phys. Rev. Lett. 90, 082302 (2003)

6. J. Adams et al. (STAR Collaboration), Phys. Rev. Lett. 97, 162301 (2006)

7. N. Armesto, L. Cunqueiro, C. Salgado, J. High Energy Phys. 2, 48 (2008)

8. R. Baier et al., Nucl. Phys. B 484, 265 (1997)

9. A. Dainese, C. Loizides, G. Paic, Eur. Phys. J. C 38, 461 (2005)

10. C.A. Salgado, U.A. Wiedemann, Phys. Rev. Lett. 93, 042301 (2004)

11. D.E. Kharzeev, arXiv:0806.0358 [hep-ph]

12. I.P. Lokhtin, S.V. Petrushanko, A.M. Snigirev, C.Y. Teplov, PoS LHC07, 003 (2007)

13. A. Accardi, N. Armesto, I.P. Lokhtin, hep-ph/0211314 (2002)

14. N. Grau (ATLAS Collaboration), J. Phys. G 35, 104040 (2008)

15. ATLAS Collaboration, ATLAS Technical Design Report, vol. 1, CERN-LHCC-99-14, 1999

16. ATLAS Collaboration, ATLAS Technical Design Report, vol. 2, CERN-LHCC-99-15, 1999

17. ATLAS Collaboration, Heavy Ion Physics with the ATLAS Detector, CERN-LHCC-2004-009 I-013, 2004

18. T. Sjostrand, S. Mrenna, P. Skands, J. High Energy Phys. 0605, 026 (2006)

19. M. Gyulassy, X.-N. Wang, Comput. Phys. Commun. 83, 307 (1984)

20. G. Sterman, S. Weinberg, Phys. Rev. Lett. 39, 1436 (1977)

21. R. Akers et al. (OPAL Collaboration), Z. Phys. C 63, 197 (1994)

22. I. Vitev, arXiv:0806.0003 [hep-ph]

23. I.P. Lokhtin, A.M. Snigirev, Eur. Phys. J. C 46, 211 (2006) 